Copyright (C) 2014 by Academic Publishing House Researcher

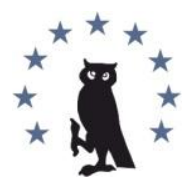

Published in the Russian Federation

European Researcher

Has been issued since 2010.

ISSN 2219-8229

E-ISSN 2224-0136

Vol. 79, No. 7-2, pp. 1296-1300, 2014

DOI: 10.13187/issn.2219-8229

www.erjournal.ru



Physical and Mathematical sciences

Физико-Математические науки

\title{
The First Main Boundary Value Problem of Dynamics of Thermo-Resiliency's Momentum Theory
}

\author{
${ }^{1}$ Merab Aghniashvili \\ 2 Diana Mtchedlishvili
}

1-2 Iakob Gogebashvili Telavi State University, Georgia

2200 Telavi, 1, Kartuli Universiteti Str.

Doctor of Physical and Mathematical sciences

E-mail: diana.mtchedlishvili@gmail.com

${ }^{1}$ Associate Professor

${ }^{2}$ Professor

\section{Abstract}

In the paper, the first main boundary value problem of Dynamics of Thermo-resiliency's momentum theory is converted into the Elliptic boundary value problem with the formal usage of the Laplace transform. This problem is studied using the method of singular integral equations. The solution of the first main boundary value problem is derived using the inverse Laplace transform.

Keywords: the first main boundary value problem; Elliptic boundary value problem; Laplace transform; inverse Laplace transform; singular integral equation.

\section{Introduction}

Let $D$ be a finite or infinite three-dimensional space with the compact boundary $S$ from the class $\Lambda_{2}(\alpha),(\alpha>0)$.

Denote by $D_{l}$ and $S_{l}$ cylinders $D_{l}=D \times l, S_{l}=S \times l$, respectively, where $l=[0, \infty)$.

The main equations of the thermo-resiliency's momentum theory can be written in a vector form as follows [1], [2]:

$$
\begin{gathered}
(\mu+\alpha) \Delta u+(\lambda+\mu-\alpha) \text { grad div } u+2 \alpha \operatorname{rot} \omega-v \operatorname{grad} \theta+\rho F=\rho \partial_{t}^{2} u, \\
(\gamma+\varepsilon) \Delta \omega+(\beta+\gamma-\varepsilon) \operatorname{grad} \operatorname{div} \omega-4 \alpha \omega+2 \alpha \operatorname{rot} u+\rho \mathcal{Y}=\zeta \partial_{t}^{2} \omega, \\
\Delta \theta-\frac{1}{\vartheta} \partial_{t} \theta-\eta \partial_{t} \operatorname{div} u+\frac{1}{\vartheta} \mathbb{Q}=0,
\end{gathered}
$$

where $u(x, t)=\left(u_{1}, u_{2}, u_{3}\right)$ is a movement vector, while $\omega(x, t)=\left(\omega_{1}, \omega_{2}, \omega_{3}\right)$ is a rotation vector and $\theta(x, t)$ - temperature. By $\rho, \lambda, \mu, \alpha, \beta, \gamma, \varepsilon, \zeta, \nu, \vartheta, \eta$ are denoted resiliency constants, by $F=$ 
$\left(F_{1}, F_{2}, F_{3}\right), \mathcal{Y}=\left(\mathcal{Y}_{1}, \mathcal{Y}_{2}, \mathcal{Y}_{3}\right), Q$ - weight power, weight moment and temperature source, respectively.

The main equations of the thermo-resiliency's momentum theory can be written as:

$$
M\left(\partial_{x}\right) U-v \chi \theta-\chi^{0} \frac{\partial^{2} u}{\partial t^{2}}=\mathcal{H}, \Delta \theta-\frac{1}{\vartheta} \frac{\partial \theta}{\partial t}-\eta \frac{\partial}{\partial t} \operatorname{div} u=\mathcal{H}_{7}
$$

where $M\left(\partial_{x}\right)$ is a differential operator of the momentum resilience theory [3] and $\chi=\left(\partial_{x_{1}}, \partial_{x_{2}}, \partial_{x_{3}}\right), \chi^{0}=\left\|\chi_{i j}\right\|_{6 \times 6}, \chi_{i i}^{0}=\rho$ for $i=1,2,3, \chi_{i i}^{0}=\zeta$ for $i=4,5,6, \chi_{i j}^{0}=0$ for $i \neq j$, $\mathcal{H}=(-\rho F,-\rho \mathcal{Y}), \mathcal{H}_{7}=-\frac{1}{\vartheta} Q, \mathcal{U}=(u, v)$.

The first problem states: to find in the cylinder $D_{l}$ the solution $U=(\mathcal{U}, \theta)$ of the equation (1) belonging to $C^{1}\left(\bar{D}_{l}\right) \cap C^{2}\left(D_{l}\right)$ and satisfying the initial and boundary conditions:

$$
\begin{gathered}
\lim _{t \rightarrow 0} U(x, t)=\varphi^{(0)}(x), \lim _{t \rightarrow 0} \theta(x, t)=\varphi_{7}^{(0)}(x), \lim _{t \rightarrow 0} \frac{\partial u(x, t)}{\partial t}=\varphi^{(1)}(x), \\
\lim _{D \ni x \rightarrow y \in S} U(x, t)=f(y, t), \lim _{D \ni x \rightarrow y \in S} \theta(x, t)=f_{7}(y, t),
\end{gathered}
$$

where $\varphi^{(i)}=\left({ }_{\varphi}^{1}(i),{ }_{\varphi}^{2}(i)\right)$ for $i=0,1,{ }_{\varphi}^{k}(i)=\left(\varphi_{1}^{k_{(i)}}, \varphi_{2}^{k_{(i)}}, \varphi_{3}^{k_{(i)}}\right)$ for $k=1,2$ and $\varphi_{7}^{(i)}$ for $i=0,1$ are functions given in the area $\bar{D}$, while $f=\left(f^{(1)}, f^{(2)}\right), f^{(i)}=\left(f_{1}^{(i)}, f_{2}^{(i)}, f_{3}^{(i)}\right)$ for $i=1,2$ and $f_{7}$ are functions given on $S_{l}$.

The uniqueness theorem of the solution was proved for this problem in [4].

It should be mentioned that in boundary cases some conditions are required to be fulfilled [5].

\section{Solution of the problem}

The stated problem is converted into the Elliptic problem as follows below.

Consider the vector function

$$
H=\left(h ; h_{7}\right), h=\left(h^{(1)}, h^{(2)}\right), h^{(i)}=\left(h_{1}^{(i)}, h_{2}^{(i)}, h_{3}^{(i)}\right) \text { for } i=1,2,
$$

where $h=e^{-t^{7}} \sum_{k=0}^{6} \frac{t^{k}}{k !} \varphi^{(k)}(x), h_{7}=e^{-t^{5}} \sum_{k=0}^{4} \frac{t^{k}}{k !} \varphi_{7}^{(k)}(x)$.

Let $U=(\mathcal{U}, \theta)$ be a solution of the first problem, then $U_{0}=U-H$ will be a solution of the following problem:

where

$$
\begin{gathered}
M\left(\partial_{x}\right) u_{0}-v \chi \theta_{0}-\chi^{0} \frac{\partial^{2} u_{0}}{\partial t^{2}}=\mathcal{H}_{0}, \\
\Delta \theta_{0}-\frac{1}{\vartheta} \frac{\partial \theta_{0}}{\partial t}-\eta \frac{\partial}{\partial t} \operatorname{div} u_{0}=\mathcal{H}_{07}, \\
\lim _{t \rightarrow 0} u_{0}(x, t)=0, \lim _{t \rightarrow 0} \frac{\partial u_{0}(x, t)}{\partial t}=0, \\
\lim _{D \ni x \rightarrow y \in S} u_{0}(x, t)=f(y, t)-h(y, t) \equiv f_{0}(y, t), \\
\lim _{D \ni x \rightarrow y \in S} \theta_{0}(x, t)=f_{7}(y, t)-h_{7}(y, t) \equiv f_{07}(y, t),
\end{gathered}
$$

$$
\begin{gathered}
\mathcal{H}_{0}=\mathcal{H}-M\left(\partial_{x}\right) h+v \chi \mathrm{h}_{7}-\chi^{0} \frac{\partial^{2} h}{\partial t^{2}} \\
\mathcal{H}_{07}=\mathcal{H}_{7}-\Delta \mathrm{h}_{7}+\frac{1}{\vartheta} \frac{\partial \mathrm{h}_{7}}{\partial t}-\eta \frac{\partial}{\partial t} \operatorname{div} h^{(1)}
\end{gathered}
$$

From (9) and (10) we can derive that

$$
\left(\frac{\partial^{m} \mathcal{H}_{0}}{\partial t^{m}}\right)_{t=0}=0,\left(\frac{\partial^{k} \mathcal{H}_{07}}{\partial t^{k}}\right)_{t=0}=0, x \in \bar{D} \text { for } m=0,1,2,3,4 \text { and } \mathrm{k}=0,1,2,3 \text {. }
$$

Analogously, from (7) and (8) we get that

$$
\left(\frac{\partial^{m} f_{0}(y, t)}{\partial t^{m}}\right)_{t=0}=0,\left(\frac{\partial^{k} f_{07}(y, t)}{\partial t^{k}}\right)_{t=0}=0, y \in S \text { for } m=0, \ldots, 6 \text { and } \mathrm{k}=0, \ldots, 4 \text {. }
$$

Let $\tau=\varsigma+i \xi$ be a complex variable and $\varsigma>\varsigma^{\prime}$, where $\varsigma^{\prime}$ is a constant from [5] (condition $4_{0}$ ). We have:

$$
\begin{gathered}
\widetilde{\mathcal{H}_{0}}(x, \tau)=\int_{0}^{\infty} e^{-\tau t} \mathcal{H}_{0}(x, t) d t, \widetilde{\mathcal{H}_{07}}(x, \tau)=\int_{0}^{\infty} e^{-\tau t} \mathcal{H}_{07}(x, t) d t \\
\widetilde{f_{0}}(y, \tau)=\int_{0}^{\infty} e^{-\tau t} f_{0}(y, t) d t, \widetilde{f_{07}}(y, \tau)=\int_{0}^{\infty} e^{-\tau t} f_{07}(y, t) d t .
\end{gathered}
$$

$\mathcal{H}, \mathcal{H}_{7}, f, f_{7}, \varphi^{(0)}, \varphi^{(1)}, \varphi_{7}^{0}$ are taken from [5] according to $1_{0}-5_{0}$ conditions, where $\varsigma>\varsigma^{\prime}$ and integrals (13) and (14) are absolutely and uniformly convergent.

With the formal usage of the Laplace transform

$$
\widetilde{U_{0}}(\mathrm{x}, \tau)=\int_{0}^{\infty} e^{-\tau t} U_{0}(x, t) d t
$$

the stated boundary value problem can be converted into the following boundary value problem[6]: 


$$
\begin{gathered}
M\left(\partial_{x}\right) \widetilde{u_{0}}(x, \tau)-\chi^{0} \tau^{2} \widetilde{u_{0}}(x, \tau)-v \chi \widetilde{\theta_{0}}(x, \tau)=\widetilde{\mathcal{H}_{0}}(x, \tau), \\
\Delta \widetilde{\theta_{0}}(x, \tau)-\frac{\tau}{\vartheta} \widetilde{\theta_{0}}(x, \tau)-\eta \tau \operatorname{div} \widetilde{u_{0}}(x, \tau)=\widetilde{\mathcal{H}_{07}}(x, \tau), \\
\lim _{D \ni x \rightarrow y \in S} \widetilde{u_{0}}(x, \tau)=\widetilde{f_{0}}(y, \tau), \lim _{D \ni x \rightarrow y \in S} \widetilde{\theta_{0}}(x, \tau)=\widetilde{f_{07}}(y, \tau) .
\end{gathered}
$$

Let us denote this problem by $\left(I_{\tau}\right)$.

The existence and uniqueness theorem of this problem is given in [7].

In order to have the inverse Laplace transform for the function $\widetilde{U_{0}}(\mathrm{x}, \tau)$ and this transform would give the classical solution of the problem $\left(I_{\tau}\right)$, we should prove some estimates for the function $\widetilde{U_{0}}(\mathrm{x}, \tau)$ and for its up to the second derivatives with respect to $\tau$.

For this purpose, we will present the solution of the problem $\left(I_{\tau}\right)$ by the sum of the solutions of the following problems:

$$
\begin{aligned}
& M\left(\partial_{x}\right) \widetilde{u_{0}^{(1)}}(x, \tau)-\chi^{0} \tau^{2} \widetilde{u_{0}^{(1)}}(x, \tau)=0, \\
& \Delta{\widetilde{\theta_{0}}}^{(1)}(x, \tau)-\frac{\tau}{\vartheta} \widetilde{\theta}_{0}^{(1)}(x, \tau)=0, \\
& \lim _{D \ni x \rightarrow y \in S} \widetilde{u_{0}^{(1)}}(x, \tau)=\widetilde{f}_{0}(y, \tau), \\
& \lim _{D \ni x \rightarrow y \in S} \widetilde{\theta_{0}}(1)(x, \tau)=\widetilde{f_{07}}(y, \tau), \\
& M\left(\partial_{x}\right) \widetilde{u_{0}^{(2)}}(x, \tau)-\chi^{0} \tau^{2} \widetilde{{u_{0}}^{(2)}}(x, \tau)-v \chi{\widetilde{\theta_{0}}}^{(2)}(x, \tau)=\widetilde{\mathcal{H}_{0}^{(1)}}(x, \tau) \text {, } \\
& \Delta{\widetilde{\theta_{0}}}^{(2)}(x, \tau)-\frac{\tau}{\vartheta}{\widetilde{\theta_{0}}}^{(2)}(x, \tau)-\eta \tau \operatorname{div} \widetilde{u_{0}}{ }^{(2)}(x, \tau)=\widetilde{\mathcal{H}_{07}{ }^{(1)}}(x, \tau), \\
& \lim _{D \ni x \rightarrow y \in S} \widetilde{u_{0}^{(2)}}(x, \tau)=0 .
\end{aligned}
$$

Denote that $\widetilde{U_{0}^{(1)}}=\left(\widetilde{u_{0}^{(1)}},{\widetilde{\theta_{0}}}^{(1)}\right)$ and $\widetilde{U_{0}^{(2)}}=\left(\widetilde{u_{0}^{(2)}}, \widetilde{\theta_{0}}{ }^{(2)}\right)$.

Let us show that $\widetilde{U_{0}{ }^{(1)}}(x, \tau)$ and $\widetilde{U_{0}{ }^{(2)}}(x, \tau)$ are analytic functions with respect to $\tau$ and determine asymptotic assessments of these functions and their derivatives.

At first we will begin with the function $\widehat{U_{0}^{(1)}}(x, \tau)$. Using the method of partial integration for the integrals from (13) and (14), according to (11) and (12), and considering the conditions $1_{0}-5_{0}$ from [5], we get:

$$
\begin{gathered}
\widetilde{\mathcal{H}_{0}}(x, \tau) \in C^{1, \delta}(\bar{D}), \widetilde{\mathcal{H}_{07}}(x, \tau) \in C^{1, \delta}(\bar{D}), \\
\widetilde{f_{0}}(y, \tau) \in C^{1, \lambda}(S), \widetilde{f_{07}}(y, \tau) \in C^{1, \lambda}(S) .
\end{gathered}
$$

Then, in the half-plane $\Pi_{S_{0}}$ the following assessments take place:

$$
\begin{aligned}
\left\|\widetilde{\mathcal{H}_{0}}(x, \tau)\right\|_{(\bar{D}, 0, \delta)} & \leq \frac{c}{|\tau|^{6}},\left\|\widetilde{\mathcal{H}_{07}}(x, \tau)\right\|_{(\bar{D}, 0, \delta)} \leq \frac{c}{|\tau|^{5}}, \\
\left\|\widetilde{f}_{0}(y, \tau)\right\|_{(S, 0, \delta)} & \leq \frac{c}{|\tau|^{8}},\left\|\widetilde{f_{07}}(y, \tau)\right\|_{(S, 0, \delta)} \leq \frac{c}{|\tau|^{6}}, \\
\left\|\widetilde{f}_{0}(y, \tau)\right\|_{(S, 1, \delta)} & \leq \frac{c}{|\tau|^{7}},\left\|\widetilde{f_{07}}(y, \tau)\right\|_{(S, 1, \delta)} \leq \frac{c}{|\tau|^{6}}
\end{aligned}
$$

According to $5_{0}$ from [5], for the rather high value of $|x|$, we will have:

$$
\left|\widetilde{\mathcal{H}_{0}}(x, \tau)\right| \leq \frac{c}{|x|^{2}} \frac{1}{|\tau|^{6}},\left|\widetilde{\mathcal{H}_{07}}(x, \tau)\right| \leq \frac{c}{|x|^{2}} \frac{1}{|\tau|^{5}} .
$$

The problems (16) and (18) have the unique solution [8] and can be given as

$$
\left.u_{0}{ }^{(1)}(x, \tau)=\int_{S} \llbracket T\left(\partial_{y}, n(y)\right) \Psi^{\prime}(\mathrm{z}-\mathrm{y} ; \mathrm{i} \tau) \rrbracket '\right\}(y, \tau) d_{y} S,
$$

where $T\left(\partial_{y}, n(y)\right)$ is an operator of momentary voltage and $\Psi(\mathrm{z}-\mathrm{y} ; \mathrm{i} \tau)-$ a matrix of the fundamental solutions of the equation (16) [3].

In case of internal problem the vector-function $\psi(y, \tau)$ represents the solution of the integral equation:

$$
-\psi(z, \tau)+\int_{S} \llbracket T\left(\partial_{y}, n(y)\right) \Psi^{\prime}(\mathrm{z}-\mathrm{y} ; \mathrm{i} \tau) \rrbracket^{\prime} \psi(y, \tau) d_{y} S=\widetilde{f}_{0}(z, \tau) .
$$

And in case of external problem it represents the solution of the integral equation:

$$
\psi(z, \tau)+\int_{S} \llbracket T\left(\partial_{y}, n(y)\right) \Psi^{\prime}(\mathrm{z}-\mathrm{y} ; \mathrm{i} \tau) \rrbracket^{\prime} \psi(y, \tau) d_{y} S=\widetilde{f}_{0}(z, \tau) .
$$

In the half-plane $\Pi_{S_{0}}$ the solutions of these equations are analytic with respect to $\tau$, as the right sides of these equations are analytic functions with respect to $\tau$. Hence, $\mathcal{U}_{0}{ }^{(1)}(x, \tau)$ is also an analytic function with respect to $\tau$. 
According to the Banach theorem, the operators corresponding to the equations (30) and (31) have inverse operators in the class $C^{0, \lambda}(S)$. Hence, we have:

from which, according to (26) we get:

$$
\|\psi(\cdot, \tau)\|_{(S, 0, \lambda)} \leq C\left\|\widetilde{f}_{0}(\cdot, \tau)\right\|_{(S, 0, \lambda)},
$$

Analogously, from (27) we have:

$$
\|\psi(\cdot, \tau)\|_{(S, 0, \lambda)} \leq \frac{c}{|\tau|^{8}} .
$$

According to (32) and (33), from (27) follows that

$$
\|\psi(\cdot, \tau)\|_{(S, 1, \lambda)} \leq \frac{c}{|\tau|^{7}} .
$$

In case of external problem we will have:



$$
\left|\widetilde{u_{0}^{(1)}}(x, \tau)\right| \leq \frac{c}{|x|^{2}} \frac{1}{|\tau|^{8}},\left|\frac{\partial}{\partial x_{k}} \widetilde{u_{0}^{(1)}}(x, \tau)\right| \leq \frac{c}{|x|^{2}} \frac{1}{|\tau|^{8}},
$$

where $k=1,2,3$ and $\tau \in \Pi_{S_{0}}$.

For each $\bar{D}^{*} \subset D$ takes place the assessment:

$$
\sup _{x \in D^{*}}\left|\frac{\partial^{2}}{\partial x_{k} \partial x_{j}} \widetilde{u_{0}^{(1)}}(x, \tau)\right| \leq \frac{C\left(D^{*}\right)}{|\tau|^{8}}, \tau \in \Pi_{\varsigma_{0}} .
$$

There exists a solution of the problem (17) and (19), it is unique and can be expressed as [3]:

$$
{\widetilde{\theta_{0}}}^{(1)}(x, \tau)=\int_{S} \frac{\partial}{\partial n(y)} \frac{e^{-k|x-y|}}{|x-y|} \varphi_{7}(y, \tau) d_{y} S, x \in D,
$$

where $\varphi_{7}(x, \tau)$ represents a solution of the singular equation:

$$
\pm \varphi(z, \tau)+\frac{1}{2 \pi} \int_{S} \frac{\partial}{\partial n(y)} \frac{e^{-k|z-y|}}{|z-y|} \varphi_{7}(y, \tau) d_{y} S=\frac{1}{2 \pi} \widetilde{f_{07}}(z, \tau),
$$

for the external and internal problems, respectively.

As the function $\widetilde{f_{07}}(z, \tau)$ is analytic with respect to $\tau$, then the solution of the equation (37) is also analytic with respect to $\tau$. Hence, ${\widetilde{\theta_{0}}}^{(1)}(x, \tau)$ will be analytic with respect to $\tau$, as well.

For each $\tau \in \Pi_{S_{0}}$, according to (26) and (27) and using the Banach theorem, from (37) we will have:

Hence, we derive that

$$
\left\|\varphi_{7}(\cdot, \tau)\right\|_{(S, 0, \lambda)}<\frac{c}{|\tau|^{6}},\left\|\varphi_{7}(\cdot, \tau)\right\|_{(S, 1, \lambda)}<\frac{c}{|\tau|^{5}} .
$$

$$
\begin{gathered}
\left\|\widetilde{\theta_{0}^{(1)}}(\cdot, \tau)\right\|_{\left(\bar{D}^{+}, 0, \lambda\right)} \leq \frac{c}{|\tau|^{6}},\left\|\widetilde{\theta_{0}^{(1)}}(\cdot, \tau)\right\|_{\left(\bar{D}^{+}, 1, \lambda\right)} \leq \frac{c}{|\tau|^{5}}, \\
\left|\frac{\partial}{\partial x_{k}} \widetilde{\theta_{0}^{(1)}}(x, \tau)\right| \leq \frac{c}{|\tau|^{3}}, x \in \bar{D}^{+}, \mathrm{k}=1,2,3 .
\end{gathered}
$$

For the highest value of $|x|$ we will have:

$$
\begin{gathered}
\left|\widetilde{\theta_{0}^{(1)}}(x, \tau)\right| \leq \frac{c}{|x|^{2}} \frac{1}{|\tau|^{6}},\left|\frac{\partial}{\partial x_{k}} \widetilde{\theta_{0}^{(1)}}(x, \tau)\right| \leq \frac{c}{|x|^{2}} \frac{1}{|\tau|^{5}}, \mathrm{k}=1,2,3 . \\
\sup _{x \in D^{*}}\left|\frac{\partial^{2}}{\partial x_{k} \partial x_{j}} \widetilde{\theta_{0}^{(1)}}(x, \tau)\right| \leq \frac{c}{|\tau|^{6}}, k, j=1,2,3 .
\end{gathered}
$$

From (34), (35) and (39) follows that

$$
\left|\widetilde{U_{0}^{(1)}}(x, \tau)\right| \leq \frac{c}{|\tau|^{5}} \text {. }
$$

The following assessments are true:

From (41) and (42) we get that

$$
\left|\widetilde{U_{0}^{(2)}}(x, \tau)\right| \leq \frac{c}{|\tau|^{4}}, x \in \bar{D}
$$

$$
\left|\widetilde{U_{0}}(x, \tau)\right| \leq \frac{c}{|\tau|^{4}} .
$$

The auxiliary statement. The following theorem is true [6]:

Theorem. Let $\tau \in \Pi_{S_{0}}$, then the problem (I) $\tau$ has the unique, analytic solution $\widetilde{U_{0}}(x, \tau)$ with respect to $\tau$, for which the following assessments take place:
1) $\left|\widetilde{U_{0}}(x, \tau)\right| \leq \frac{c}{|\tau|^{4}},\left|\frac{\partial \widetilde{U_{0}(x, \tau)}}{\partial x_{k}}\right| \leq \frac{c}{|\tau|^{2+\frac{2}{3}}}$ for any $(x, \tau) \in \bar{D} \times \Pi_{\zeta_{0}}$;
2) $\left|\frac{\partial^{2} \widetilde{U}_{0}(x, \tau)}{\partial x_{k} \partial x_{l}}\right| \leq \frac{c}{|\tau|^{1+\frac{1}{9}}}$ for any $(x, \tau) \in \bar{D} \times \Pi_{S_{0}}$; 
3) $\left|\widetilde{U_{0}}(x, \tau)\right| \leq \frac{c}{|x|^{2}|\tau|^{2+\frac{2}{3}}},\left|\frac{\partial \widetilde{U_{0}}(x, \tau)}{\partial x_{k}}\right| \leq \frac{c}{|x|^{2}|\tau|^{1+\frac{2}{3}}}$ for $x \rightarrow \infty$, where $k, l=1,2,3$.

\section{The main result}

Using the above mentioned auxiliary theorem and based on the properties of the Laplace transform, we prove the following result:

Theorem. The first problem of the thermo-resiliency's momentum theory has in $D_{l}$ a unique solution which can be presented as:

$$
U(x, t)=H(x, t)+\frac{1}{2 \pi i} \int_{\varsigma-i \infty}^{\varsigma+i \infty} e^{\tau t} \widetilde{U_{0}}(x, \tau) d \tau, \varsigma>\varsigma_{0}^{\prime},
$$

where $\widetilde{U_{0}}(x, \tau)$ represents the solution of the problem $(\mathrm{I})_{\tau}$ and $H(x, t)$ is a vector-function $\left(^{*}\right)$.

\section{Conclusion}

The main task was to convert the first main boundary value problem of the thermoresiliency's momentum theory into the oscillatory problem using the Laplace transform. In the paper, the solution of the oscillatory problem is found and there are given those conditions, which enable to prove the existence of the inverse transform. The effective solution of the main boundary value problem is found using this inverse Laplace transform.

\section{References}

Novatski, V. (1975). Resilience theory. Moscow (in Russian);

Aghniashvili, M. (1976). Some boundary value problems of thermo-momentum resilience theory. Tbilisi (in Russian);

Kupradze, V., Gegelia, T., Basheleishvili, M., Burtchuladze, T. (1976). Three-dimensional problems of mathematical resilience theory and thermo-resiliency. Tbilisi (in Russian);

Aghniashvili, M., Mtchedlishvili, D. (2006). The statement of the main boundary problems of thermo-momentum theory of resiliency and the uniqueness theorems. Scientific Papers of I. Gogebashvili Telavi State University, 2 (20), pp. 20-21 (in Georgian);

Aghniashvili, M., (1977). Dynamical problems of momentum theory of thermo-resiliency. Bulletin of the Georgian National Academy of Sciences, 2 (86) (in Russian);

Aghniashvili, M., Mtchedlishvili, D. (2006). The reduction of the dynamics first problem of thermo-momentum theory of resiliency to the elliptic problem. Periodical Scientific Journal "Intelecti", 3 (26), pp. 11-12 (in Georgian);

Aghniashvili, M., Mtchedlishvili, D. (2006). The theorems of existence and uniqueness of the elliptic problem solution. Scientific Papers of I. Gogebashvili Telavi State University, 2 (20), pp. 22-26 (in Georgian);

Kapanadze, R., Tchitchinadze R. (1976). Some boundary value problems of dynamics of momentum resilience theory. Tbilisi (in Russian); 